EPJ Web of Conferences 82, 01031 (2015)

DOI: $10.1051 /$ epjconf/20158201031

(C) Owned by the authors, published by EDP Sciences, 2015

\title{
Forecasting investigation of mode fire hazard of electrical overload of cable lines
}

\author{
Mooza M. Grigorieva, Evgenia V. Ivanova and Pavel A. Strizhak ${ }^{\mathrm{a}}$ \\ National Research Tomsk Polytechnic University, 634050 Tomsk, Russia
}

\begin{abstract}
The physical and predictive mathematical models of heat and mass transfer, phase transformations and chemical reaction have been developed to determine the necessary and sufficient conditions of inflammation and fire nascence at typical cable lines electrical overloads. The integral characteristics of study process complex - ignition delay times have been established according to cable lines electrical overloads modes. The temperature distributions in typical cable lines at overloads have been determined. The concentration variation characteristic ranges (which are sufficient for gas-phase ignition) of thermal decomposition components of cable sheaths at overloads have been adduced. The two-dimensional heat-and-mass transfer problem for a three-layer cable in the area of limited heat sink is solved. Thermal decomposition of the cable sheath and diffusion of thermal decomposition components and air were taken into account. Fire risk assessment of cable lines on overload in conditions of limited heat transfer is carried out. The dependence of ignition time delay for mixture of oxidized and thermal decomposition components from intensity of a current.
\end{abstract}

\section{Introduction}

Electrical products are most flammable by analysis of statistical data [1]. Wires and cables hold first place for all types electrical product by the number of fires, victims and largest property damage [2-4].

Cause ignition cable is overheating of conductors due to a short circuit or overload. There is inducing igniting insulation. Concentration of cables in a confined space can result in serious damage in case of fire. Fire safety of cable lines is largely determined by the state of incorporation of cable passes from one room to another, and condition of the insulation.

To date, assessment of the fire hazard of cable articles carried on the assumption that the cross section of the conductor temperature constant. There is into account only the change in temperature of the cable in time. This approach is too simplistic and is typically used to determine the temporal characteristics of pyrolysis and ignition insulation overload cable lines. The disadvantage is described in [5] model is that it ignores the conditions when the heat exchange with the environment is difficult (cable immured in a wall or placed in a box). So far, there are no methods to predict fire hazard cable lines with limited heat and overload.

\footnotetext{
${ }^{a}$ Corresponding author: pavelspa@tpu.ru
}

This is an Open Access article distributed under the terms of the Creative Commons Attribution License 4.0, which permits unrestricted use, distribution, and reproduction in any medium, provided the original work is properly cited. 


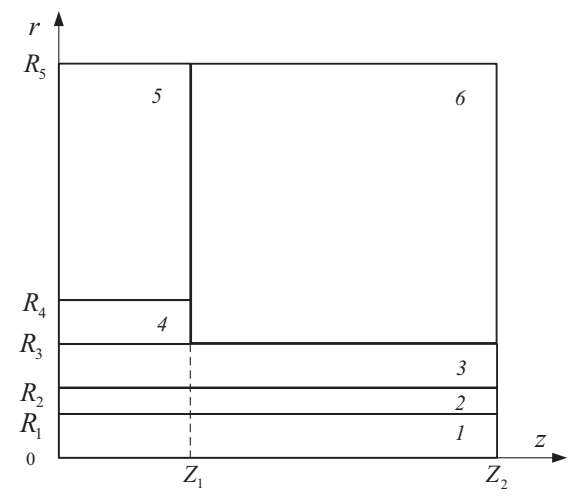

Figure 1. Scheme area of solving the problem: 1 - conductor; 2 - insulation; 3 - cable sheath; 4 - cable penetrations; 5 - bulkhead (wall); 6 - oxidant mixture with components of thermal decomposition of shell materials and cable insulation.

The aim of this work is to assess the fire hazard regimes overload of cable lines with limited heat transfer on the basis of mathematical modeling of unsteady temperature fields.

\section{Problem statement}

There are considered solid cable with insulation and sheath. Choice due to the fact that having multiple cores may not significantly affect the temperature change due to the high thermal conductivity of metals from which they are made. Multilayer insulation can be represented by a single layer in solving the problem, since most insulation materials have similar thermal characteristics meaningfully. There are accepted that cable runs from one room to another, so part of it is immured in the wall (septum) using termination material (sealant). The geometry of the domain solution of the problem is shown in Fig. 1.

There are considered the following diagram of the process. The conductor was heated to a temperature exceeding the permissible when electrical overload. There are occurs heating cable sheath material and sealant cable insulation. At a certain temperature $T_{p}$, characteristic for each material and close to its ignition temperature $T_{v}$, chemical reactions, thermal decomposition of the material the cable sheath and cable insulation sealant flow intensively. The products of thermal decomposition are mixed with air and heated by the heat liberated. Upon reaching the critical temperature and the concentration of the thermal decomposition products in the air forming mixture ignites.

\section{Mathematical model}

The mathematical model corresponding to the physical formulation of the problem formulated includes a system of differential equations of unsteady heat conduction for each layer of the system (Figure 1), the energy equation for a mixture of oxidant components of thermal decomposition, the diffusion equation of thermal decomposition of components in the medium oxidant.

Since in most cases the cables are multi-layer cylinder, the system of heat conduction equations are written in cylindrical coordinates. In the energy equation for the heat conductor is considered caused by electrical overload and changes in the electrical resistance of the material core temperature change. 
The energy equation for the core:

$$
\begin{aligned}
\rho_{1} C_{1} \frac{\partial T_{1}}{\partial t}= & \lambda_{1}\left(\frac{\partial^{2} T_{1}}{\partial r^{2}}+\frac{1}{r} \frac{\partial T_{1}}{\partial r}+\frac{\partial^{2} T_{1}}{\partial z^{2}}\right)+\frac{I^{2} \gamma}{S}, \\
0 & <r<R_{1}, 0<z<Z_{2} .
\end{aligned}
$$

The heat equation for insulation:

$$
\begin{aligned}
\rho_{2} C_{2} \frac{\partial T_{2}}{\partial t}= & \lambda_{2}\left(\frac{\partial^{2} T_{2}}{\partial r^{2}}+\frac{1}{r} \frac{\partial T_{2}}{\partial r}+\frac{\partial^{2} T_{2}}{\partial z^{2}}\right) \\
& R_{1}<r<R_{2}, 0<z<Z_{2} .
\end{aligned}
$$

Thermal decomposition of the cable sheath material and sealant, which cable is fastened to the wall is described by the Arrhenius equation [6]. There are made the assumption that in the temperature range $T_{p} \ldots T_{v}$ activation energy of thermal decomposition remains unchanged. Changing the thermophysical properties of materials in the process of thermal decomposition was not considered.

The energy equation for the cable sheath:

$$
\begin{aligned}
\rho_{3} C_{3} \frac{\partial T_{3}}{\partial t}= & \lambda_{3}\left(\frac{\partial^{2} T_{3}}{\partial r^{2}}+\frac{1}{r} \frac{\partial T_{3}}{\partial r}+\frac{\partial^{2} T_{3}}{\partial z^{2}}\right)+q_{3} \rho_{3} \frac{d \varphi_{3}}{d t}, \\
& R_{2}<r<R_{3}, \quad 0<z<Z_{2}, \\
-\frac{d \varphi_{3}}{d t}= & \varphi_{3} k_{3}^{0} \exp \left(-\frac{E_{3}}{R T_{3}}\right) .
\end{aligned}
$$

The energy equation for cable insulation (sealant):

$$
\begin{aligned}
\rho_{4} C_{4} \frac{\partial T_{4}}{\partial t}= & \lambda_{4}\left(\frac{\partial^{2} T_{4}}{\partial r^{2}}+\frac{1}{r} \frac{\partial T_{4}}{\partial r}+\frac{\partial^{2} T_{4}}{\partial z^{2}}\right)+q_{4} \rho_{4} \frac{d \varphi_{4}}{d t}, \\
& R_{3}<r<R_{4}, \quad 0<z<Z_{4}, \\
& -\frac{d \varphi_{4}}{d t}=\varphi_{4} k_{4}^{0} \exp \left(-\frac{E_{4}}{R T_{4}}\right) .
\end{aligned}
$$

The heat equation for the bulkheads (walls):

$$
\begin{aligned}
\rho_{5} C_{5} \frac{\partial T_{5}}{\partial t}= & \lambda_{5}\left(\frac{\partial^{2} T_{5}}{\partial r^{2}}+\frac{1}{r} \frac{\partial T_{5}}{\partial r}+\frac{\partial^{2} T_{5}}{\partial z^{2}}\right), \\
& R_{4}<r<R_{5}, \quad 0<z<Z_{1} .
\end{aligned}
$$

The energy equation for a mixture of oxidant components of thermal decomposition:

$$
\begin{gathered}
\rho_{6} C_{6} \frac{\partial T_{6}}{\partial t}=\lambda_{6}\left(\frac{\partial^{2} T_{6}}{\partial r^{2}}+\frac{1}{r} \frac{\partial T_{6}}{\partial r}+\frac{\partial^{2} T_{6}}{\partial z^{2}}\right)+Q_{o} W_{o}, \\
R_{3}<r<R_{5}, \quad Z_{1}<z<Z_{2} .
\end{gathered}
$$

Diffusion equation for the thermal decomposition of the components in the medium oxidant:

$$
\begin{array}{r}
\rho_{6} \frac{\partial C_{g}}{\partial t}=\rho_{6} D_{6}\left(\frac{\partial^{2} C_{g}}{\partial r^{2}}+\frac{1}{r} \frac{\partial C_{g}}{\partial r}+\frac{\partial^{2} C_{g}}{\partial z^{2}}\right)-W_{o}, \\
R_{3}<r<R_{5}, \quad Z_{1}<z<Z_{2} .
\end{array}
$$

The system of Eqs. (1)-(7) is the following notation: $\rho_{i}$ - density, $\mathrm{kg} / \mathrm{m}^{3} ; \lambda_{i}$ - thermal conductivity, $\mathrm{W} / \mathrm{m} \cdot \mathrm{K} ; C_{i}-$ specific heat; $\mathrm{J} / \mathrm{kg} \cdot \mathrm{K} ; R_{i}$ - radius, $\mathrm{m} ; S$ - cross-section, $\mathrm{m}^{2} ; Z_{1}$ - wall thickness, 
$\mathrm{m} ; Z_{2}$ - cable length, $\mathrm{m} ; T_{i}$ - the current temperature value, $\mathrm{K} ; k_{i}^{0}$ - pre-exponential factor of the chemical reaction of the thermal decomposition, $1 / \mathrm{s} ; E_{i}$ - the activation energy of a chemical reaction of thermal decomposition, $\mathrm{J} / \mathrm{mol} ; q_{i}$ - heat of gasification of the material, $\mathrm{J} / \mathrm{kg} ; \varphi_{i}-$ the current value of the volume fraction of the component subjected to thermal decomposition; $Q_{o}$ - thermal effect of chemical oxidation reactions, $\mathrm{J} / \mathrm{kg} ; W_{o}$ - mass flow rate of the chemical reaction of oxidation, $\mathrm{kg} / \mathrm{m}^{3} \cdot \mathrm{s}$; $D_{6}$ - the diffusion coefficient of thermal expansion of components in the air, $\mathrm{m}^{2} / \mathrm{s}$.

Balance equation oxidizer mixture with components of thermal decomposition:

$$
C_{g}+C_{o}=1, \quad R_{3}<r<R_{5}, \quad Z_{1}<z<Z_{2},
$$

where $C_{g}$ - mass concentration of components of thermal decomposition $\left(0<C_{g}<1\right)$; $C_{o}-$ mass concentration of oxidant.

The mass flow rate of the chemical reaction of oxidation was determined from the expression [7]:

$$
W_{o}=\rho_{6} C_{o}^{n} C_{g}^{m} k_{6}^{0} \exp \left(-\frac{E_{6}}{R T_{6}}\right),
$$

where $k_{6}^{0}$ - pre-exponential factor of the chemical reaction of oxidation, $1 / \mathrm{s} ; E_{6}$ - the activation energy of chemical oxidation reaction, $\mathrm{J} / \mathrm{mol} ; n, m$ - constant chemical reaction (reaction order). There is select the most typical case - the first order $(n=m=1)$.

Initial $(t=0)$ conditions:

$$
\begin{gathered}
T=T_{0}, \quad 0<r<R_{5}, \quad 0<z<Z_{2} ; \\
\varphi=\varphi_{0}, \quad R_{2}<r<R_{3}, \quad 0<z<Z_{2}, \quad R_{3}<r<R_{4}, \quad 0<z<Z_{1} ; \\
C_{g}=0, \quad R_{3}<r<R_{5}, \quad Z_{1}<z<Z_{2} .
\end{gathered}
$$

Boundary conditions:

1. On the symmetry axis and the boundaries for all equations satisfied the condition of zero gradient of the corresponding functions:

$$
\begin{gathered}
z=0, \quad 0<r<R_{5} ; \quad z=Z_{2}, \quad 0<r<R_{5} ; \quad \frac{\partial T}{\partial z}=0 ; \\
z=Z_{2}, \quad R_{3}<r<R_{5}: \frac{\partial C_{g}}{\partial z}=0 ; \\
r=0, \quad 0<z<Z_{2} ; \quad r=R_{5}, \quad 0<z<Z_{2}: \frac{\partial T}{\partial r}=0 ; \\
r=R_{5}, \quad Z_{1}<z<Z_{2}: \frac{\partial C_{g}}{\partial r}=0 .
\end{gathered}
$$

2. Thermal interaction between the layers of the system is described by the boundary condition of fourth generation:

$$
\begin{aligned}
& r=R_{1}, \quad 0<z<Z_{2}: T_{1}=T_{2}, \quad-\lambda_{1} \frac{\partial T_{1}}{\partial r}=-\lambda_{2} \frac{\partial T_{2}}{\partial r} \\
& r=R_{2}, \quad 0<z<Z_{2}: T_{2}=T_{3}, \quad-\lambda_{2} \frac{\partial T_{2}}{\partial r}=-\lambda_{3} \frac{\partial T_{3}}{\partial r} ; \\
& r=R_{3}, \quad 0<z<Z_{1}: T_{3}=T_{4}, \quad-\lambda_{3} \frac{\partial T_{3}}{\partial r}=-\lambda_{4} \frac{\partial T_{4}}{\partial r} ; \\
& r=R_{4}, \quad 0<z<Z_{1}: T_{4}=T_{5}, \quad-\lambda_{4} \frac{\partial T_{4}}{\partial r}=-\lambda_{5} \frac{\partial T_{5}}{\partial r} .
\end{aligned}
$$


3. Since the thermal expansion does not take place only on the boundary "air-material", and the entire thickness of the degradable layer, the boundary condition on the surface of the cable and the sealant in the interface with the oxidant are described by:

$$
\begin{gathered}
r=R_{3}, \quad Z_{1}<z<Z_{2}: \\
-\lambda_{3} \frac{\partial T_{3}}{\partial r}-q_{3} \int_{r=R_{2}}^{r=R_{3}} \rho_{3} \varphi_{3} k_{3}^{0} \exp \left(-\frac{E_{3}}{R T_{3}}\right) d r=-\lambda_{6} \frac{\partial T_{6}}{\partial r}, \\
-\rho_{6} D_{6} \frac{\partial C_{g}}{\partial r}=\int_{r=R_{2}}^{r=R_{3}} \rho_{3} \varphi_{3} k_{3}^{0} \exp \left(-\frac{E_{3}}{R T_{3}}\right) d r \\
-\lambda_{4} \frac{\partial T_{4}}{\partial z}-q_{4} \int_{z=0}^{z=Z_{1}} \rho_{4} \varphi_{4} k_{4}^{0} \exp \left(-\frac{E_{4}}{R T_{4}}\right) d z=-\lambda_{6} \frac{\partial T_{6}}{\partial z}, \\
-\rho_{6} D_{6} \frac{\partial C_{g}}{\partial z}=\int_{z=0}^{z=Z_{1}} \rho_{4} \varphi_{4} k_{4}^{0} \exp \left(-\frac{E_{4}}{R T_{4}}\right) d z .
\end{gathered}
$$

4. Boundary condition on the "wall-air":

$$
\begin{gathered}
z=Z_{1}, \quad R_{4}<r<R_{5}: \\
T_{5}=T_{6}, \quad-\lambda_{5} \frac{\partial T_{5}}{\partial z}=-\lambda_{6} \frac{\partial T_{6}}{\partial z}, \frac{\partial C_{g}}{\partial z}=0 .
\end{gathered}
$$

The system of differential Eqs. (1)-(7) describing the processes of heat and mass transfer in the system, with the appropriate initial and boundary conditions is solved by finite difference method [7]. There are to solve the differential equations of difference analogues used locally one-dimensional method [7]. The system of one-dimensional differential equations is solved by the method of iterations [7] and to run using a four-point implicit finite difference scheme [7]. Time step is $\Delta t=1 \mathrm{~s}$, step to the spatial coordinates is $\Delta r=10^{-4} \mathrm{~m}, \Delta z=10^{-3} \mathrm{~m}$. Evaluation of reliability of the results in the calculations carried out a test of conservative difference schemes (estimated energy balance in the computational domain) [8-10].

\section{Results and discussion}

There is held numerical investigations for cable cross-section $0.5 \mathrm{~mm}^{2}$ copper, porcelain insulators and elastomeric membrane. Material partitions are steel. Cable penetration through which the cable is secured in a partition formed from an elastomeric material. The temperature field wall was calculated for the site, equal to four radii of cable sheathing, as at a greater distance from the cable surface temperature septum practically unchanged.

Thermophysical properties of the layers of the system: $\lambda_{1}=384 \mathrm{~W} / \mathrm{m} \cdot \mathrm{K}, \lambda_{2}=1 \mathrm{~W} / \mathrm{m} \cdot \mathrm{K}$, $\lambda_{3}=0.16 \mathrm{~W} / \mathrm{m} \cdot \mathrm{K}, \lambda_{4}=0.16 \mathrm{~W} / \mathrm{m} \cdot \mathrm{K}, \lambda_{5}=46 \mathrm{~W} / \mathrm{m} \cdot \mathrm{K}, \lambda_{6}=0.026 \mathrm{~W} / \mathrm{m} \cdot \mathrm{K}, \rho_{1}=8800 \mathrm{~kg} / \mathrm{m}^{3}$, $\rho_{2}=2300 \mathrm{~kg} / \mathrm{m}^{3}, \quad \rho_{3}=1100 \mathrm{~kg} / \mathrm{m}^{3}, \quad \rho_{4}=1100 \mathrm{~kg} / \mathrm{m}^{3}, \quad \rho_{5}=7800 \mathrm{~kg} / \mathrm{m}^{3}, \quad \rho_{6}=1.161 \mathrm{~kg} / \mathrm{m}^{3}$, 


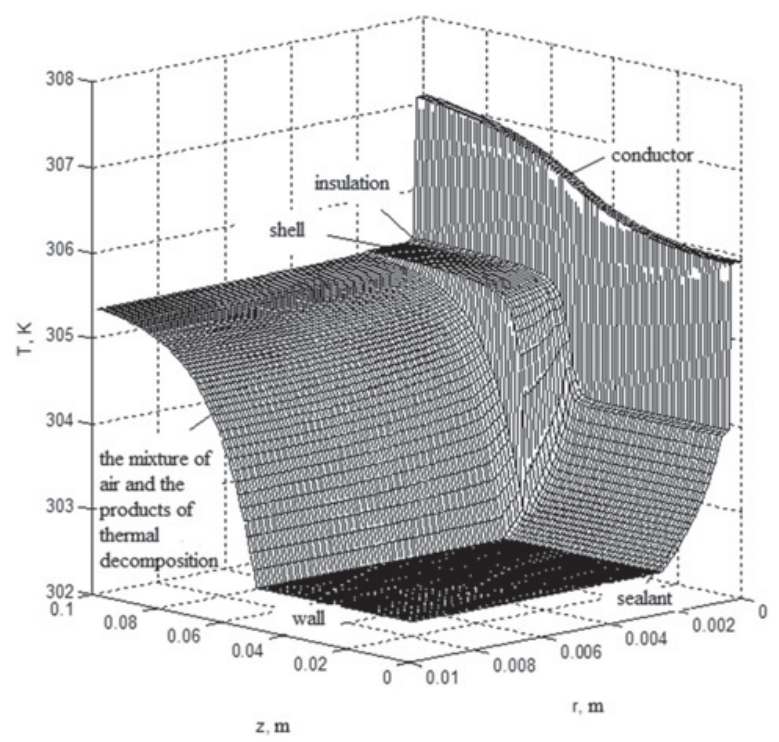

Figure 2. The temperature distribution at a current load of $10 \mathrm{~A}$.

Table 1. Maximum conductor temperature dependence of the current load.

\begin{tabular}{|l|c|c|c|c|}
\hline$I, \mathrm{~A}$ & 10 & 15 & 20 & 25 \\
\hline$T, \mathrm{~K}$ & 310 & 325 & 348 & 450 \\
\hline
\end{tabular}

Table 2. The dependence of the ignition delay time on current load.

\begin{tabular}{|c|c|c|c|c|c|}
\hline$I, \mathrm{~A}$ & 30 & 35 & 40 & 45 & 50 \\
\hline$T_{i}, \mathrm{~K}$ & 4800 & 2200 & 1300 & 500 & 100 \\
\hline
\end{tabular}

$C_{1}=381 \mathrm{~J} / \mathrm{kg} \cdot \mathrm{K}, C_{2}=1200 \mathrm{~J} / \mathrm{kg} \cdot \mathrm{K}, C_{3}=1380 \mathrm{~J} / \mathrm{kg} \cdot \mathrm{K}, C_{4}=1380 \mathrm{~J} / \mathrm{kg} \cdot \mathrm{K}, C_{5}=460 \mathrm{~J} / \mathrm{kg} \cdot \mathrm{K}$, $C_{6}=1190 \mathrm{~J} / \mathrm{kg} \cdot \mathrm{K}, D_{6}=7.83 \cdot 10^{-6} \mathrm{~m}^{2} / \mathrm{s}$.

Geometric dimensions of the cable and partitions: $R_{1}=0.0004 \mathrm{~m}, R_{2}=0.0007 \mathrm{~m}, R_{3}=0.0019 \mathrm{~m}$, $R_{4}=0.0025 \mathrm{~m}, R_{5}=0.01 \mathrm{~m}, Z_{1}=0.05 \mathrm{~m}, Z_{2}=0.25 \mathrm{~m}$.

Parameters of thermal decomposition of the shell material and sealant: $k^{0}=5 \cdot 10^{11} 1 / \mathrm{s}, q=$ $1.86 \mathrm{MJ} / \mathrm{kg}, \varphi_{0}=0.4, R=8.31 \mathrm{~J} / \mathrm{mol} \cdot \mathrm{K}, E=170 \mathrm{~kJ} / \mathrm{mol}$.

The parameters of the oxidation reaction: $k^{0}=7 \cdot 10^{9} 1 / \mathrm{s}, q=45 \mathrm{MJ} / \mathrm{kg}, E=100 \mathrm{~kJ} / \mathrm{mol}$.

Conditions of heat exchange with the environment: $T_{0}=300 \mathrm{~K}, T^{e}=300 \mathrm{~K}, \alpha=5 \mathrm{~W} / \mathrm{m}^{2} \cdot \mathrm{K}$.

Problem of numerical studies under proposed mathematical model was to determine the possible ignition mixture thermal decomposition products of the shell and the typical cable sealant products at different current loads. Figure 2 shows the temperature field in the system at the current load of $10 \mathrm{~A}$.

There are found that the shape of the temperature distribution in the studied system virtually unchanged in the range of loads, which are not implemented ignition conditions. The dependence of the maximum conductor temperature at 1:00 overloads duration of the current shown in Table 1. There are revealed that the current carrying capacity of $25 \mathrm{~A}$ is a threshold as the temperature of the thermal decomposition product mixture with an oxidizing agent after $10 \mathrm{~h}$ exposure to the cable line of said load reaches the ignition conditions listed below, but wherein the gas concentration is still not high enough for implementing ignition.

Studies have shown that the load current exceeding $25 \mathrm{~A}$ temperature and concentration of the mixture of air and the products of thermal degradation reaches a critical value at which ignition occurs. 
The dependence of the ignition delay time $t_{d}$ to the current strength is shown in Table 2. Analysis of the results of numerical studies has shown that $t_{d}$ varies from 1 hour 20 minutes at $30 \mathrm{~A}$ and 1 minute at $50 \mathrm{~A}$. There are found that ignition conditions for all investigated load current values are realized almost at the same parameters - the concentration of components of thermal decomposition in the air close to 0.005 and the temperature of the mixture at about $500 \mathrm{~K}$. The maximum temperature reached in the region at a distance of several millimetres from the wall and the cable surface. There is observed a maximum concentration of combustible components.

\section{Conclusions}

Developing model can to predict the heat and mass transfer enables the implementation of ignition conditions and prevent cable lines above flammable processes in industries. At the same time it is also possible to assess the conditions of heat transfer cable lines, ventilation and electrical limit load under which the terms of fire safety. For the selected cable load capacity is $11 \mathrm{~A}$, the temperature of the heating lived long $-338 \mathrm{~K}$, in emergency mode operation (overload) $-363 \mathrm{~K}$. It was established that the maximum heating temperature of conductor at the same load was $307 \mathrm{~K}$, which is less than the limit.

This work was supported by the Russian Foundation for Basic Research (No. 14-08-00057).

\section{References}

[1] Thomas Worzyk, Submarine Power Cables: Design, Installation, Repair, Environmental Aspects (Springer Verlag Berlin, Heidelberg, 2009)

[2] Fire Safety Guidelines for Open Plant Structures in Oil, Chemical and Process Industries. HQ Singapore Civil Defence Force 2011

[3] J. Hoover, L. Caudill, Cabling. Installation and Maintenance 6, 51 (1998)

[4] P. Morgan, C. Hardy, T. Swetnum, M. Rollins, D. Long, Intern. J. Wildland Fire 10, 329 (2001)

[5] D. Ajloo, A. Sharifian, H. Behniafar, Bulletin of the Korean Chemical Society 29, 2009 (2008)

[6] D.A. Frank-Kamenetsky, Diffusion and Heat Transfer in Chemical Kinetics (Plenum, New York, 1969)

[7] A.A. Samarskii, The Theory of Difference Schemes (Marcel Dekker Inc., USA, 2001)

[8] G.V. Kuznetsov, P.A. Strizhak, Int. J. Heat Mass Tran. 53, 923 (2010)

[9] M.M. Grigoryeva, G.V. Kuznetsov, P.A. Strizhak, J. Eng. Thermophys. 20, 192 (2011)

[10] D.O. Glushkov, G.V. Kuznetsov, P.A. Strizhak, Russ. J. Phys. Chem. B 8, 196 (2014) 